\title{
Inversion of Remote Sensing Data Using Multiple Ratios of Spectral Radiation Intensities and Neural Networks
}

\author{
S. CIĘSZCZYK* \\ Institute of Electronics and Information Technology, Lublin University of Technology, \\ Nadbystrzycka 38A, 20-618 Lublin, Poland \\ (Received May 16, 2016; in final form May 23, 2017)
}

\begin{abstract}
The article presents a method for determining the content and temperature on the basis of spectra from remote measurements. The technique uses measurements of a high resolution radiation spectrum that allows the visibility of the individual rotational lines of gases such as $\mathrm{CO}_{2}$, used here in the range of $2470-2495 \mathrm{~cm}^{-1}$. At the same time a new algorithm is applied of pre-processing the spectrum, involving the use of multiple ratios of intensity at several wavenumbers as input to an inverse model based on neural networks. Due to it, the dimensionality of the input can significantly be reduced. Additionally, the data interpreted do not have to be measured in units of spectral radiance. Thus only the calibration of the sensitivity of the spectrometer at various wavelengths is required. The neural models were constructed on the basis of data from the simulation. The proposed method works with a uniform layer of radiating gas for determining the temperature and $\mathrm{CO}_{2}$ content. For a non-uniform layer it is possible to determine the line-of-sight temperature profile and average gas content. The method can be extended to different spectral ranges and to other gases present in substantial quantities in the exhaust gases of various processes.
\end{abstract}

DOI: 10.12693/APhysPolA.131.1454

PACS/topics: remote sensing, infrared spectrometers, infrared spectra, computer modelling and simulation

\section{Introduction}

Many different processes are carried out at high temperatures. Obtaining information about their condition on a continuous basis may require sophisticated measurement techniques. If the analysed process involves emission of hot gases, it can be monitored with the use of spectral techniques. Spectral measurement can be made by active or passive methods [1], which leads to the measurement of, respectively, absorption and emission spectra. In the passive approach the observed gas layer may have a temperature higher than the background, which allows the measurement of the emission spectrum. In some cases, the background has a higher temperature than that of observed gases, with the result that the measured spectrum is an absorption one [2]. Analysis of the radiation spectra of hot gases can be used to determine their temperature and content [3-5]. This can be important information, which is not possible to determine by other methods. Radiation monitoring can be easily made from a long distance. However, the measurements must be made at a sufficiently high spectral resolution to make the spectral characteristics of the individual gases visible.

However, there appears here a problem of the very interpretation of the spectrum. To measure the temperature, typical absorption spectroscopy uses from two to several lines measured by using a laser $[6,7]$. For measurement purposes different ranges of the $\mathrm{H}_{2} \mathrm{O}[8,9]$,

*e-mail: s.cieszczyk@pollub.pl and $\mathrm{CO}_{2}$ spectrum $[10,11]$ are often used. Absorption thermometry usually measures the mean line-ofsight temperature. Recently, attempts have been undertaken to measure the temperature distribution preceded by the optimisation of the selection of the spectral lines analysed [12].

For measurements using spectroscopes, spectrum analysis it is often a complex multidimensional data analysis problem. In classical laboratory spectroscopy a calibration process is carried out using reference samples. After the calibration measurements a calibration model is constructed, which is then used for determining the content of the tested substances. This method can also be used to determine temperature, but only to a very limited extent [13]. Conditions applicable to the empirical calibration measurements in emission spectroscopy are achieved only for very short measurement paths [14]. The only problem with this type of methods is sometimes the removal of the background radiation [15]. Using the equation of radiative energy transfer, gas spectra can be calculated for specific content and temperature along the observation path [16]. The correctness of modelling methods using databases of spectral parameters of gases was confirmed in experimental conditions [17, 18]. Simulation allows to select arbitrary physical conditions of the gas layer (gas plume). In addition, one can analyse the impact of changes in various physical parameters on the radiation spectrum, that is to perform sensitivity studies. The impact of individual model parameters are studied in various types of inverse problems [19-21]. Quantitative interpretation of the measured spectrum usually consists of solving the inverse problem in relation to the problem of the calculation of the radiation spectrum, based on the knowledge of the gas contents and the temperature 
profile along the line of sight. The inverse problem can be solved in two ways [22]. The first consists in comparing the measured spectrum with the spectrum of the radiation model, which usually means a significant number of iterations. In addition, it also requires significant expertise from the person interpreting the measurement. This is due to the fact that the iterative procedure does not always give a correct result, and is dependent on the starting point of the iteration. The second method is the inverse function approximation using statistical methods. The development of such methods is associated with so-called software sensors (soft sensor, inferential measurements) or algorithms that use information from the hardware sensors measuring other physical quantities to estimate physical quantities directly not available. Sensors based on chemometric algorithms such as partial least square (PLS) or principal component regression (PCR) are data-based sensors [23]. These methods are particularly well suited to the multidimensional and correlated input data. Other commonly used methods to create data-based models include: neural networks [24] and support vector machines. At the same time, in the inverse problem various intelligent techniques [25] rather than the classic regression techniques are used. Neural networks are applied to build inverse models in various fields of physics [26-28]. The use of neural networks in spectrum analysis often requires reducing the size of the input. Various methods are used here, such as principal component analysis (PCA) [29] or wavelet transform. These methods are designed to reduce the dimensionality of the network input vector, without reducing at the same time the information contained in the data. A general disadvantage of statistical methods for reducing the dimension of the data is that they do not take into account the specific physical properties of the analysed objects. Therefore, in this study the reduction in size and the preparation of data for inverse models were based on empirical analysis of a large number of simulation spectra. Such studies provide an important knowledge base for data inversion and are carried out for different types of inverse analysis $[30,31]$. In this article, the method of intensity ratios is used, which is mainly applied in pyrometry [32] and in determining temperature based on laser absorption measurements [33]. It has so far not been used to the interpretation of passive remote sensing.

The remainder of the article is organised as follows. The next section describes the inverse problem in remote spectral measurements and the methods of solving it, including modelling of the spectra and their interpretation. Section 3 presents the results of the determination of the sought-for quantities. Conclusions are presented in Sect. 4.

\section{The inverse problem in the interpretation of hot gas radiation}

The inverse problem can be understood as inferencing about an object specific properties on the basis of observation. The direct problem is described as determination of data that can be recorded and indirectly reflect the object properties. The inverse problem appears frequently in indirect measurements, where it is not possible to directly determine the sought quantities [34]. Adequate mathematical models are then needed of the physical phenomena under consideration [35-37]. Such models can be created on the basis of physical theories or be based on measured data. In the first case they are called knowledge-driven, physical-based or mechanisticbased, to emphasise that knowledge about the phenomenon analysed is necessary here [38]. The knowledge contained in the theoretical models is called domain knowledge, because depending on the field it may be the physical, chemical or biological laws [39]. Models based on data (data-driven, back-box) do not require a detailed mathematical-physical description, but good quality data. There are also methods for combining empirical and physical models $[40,41]$.

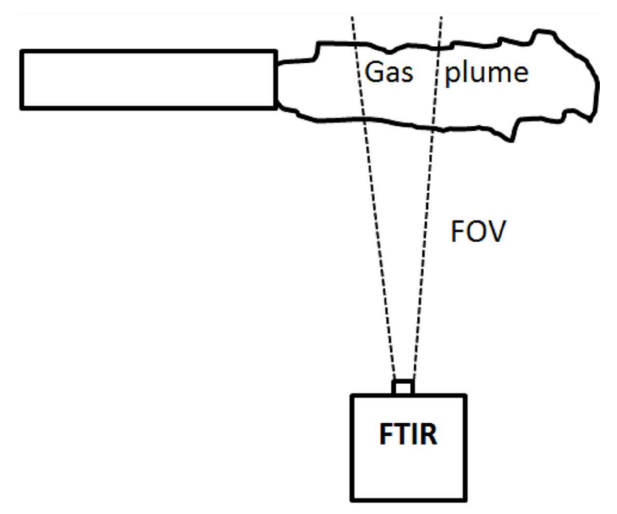

Fig. 1. Gas plume monitoring by passive spectral remote sensing.

Quantitative interpretation of remote measurements of radiation spectra is a typical example of the inverse problem in indirect measurements [42]. This technique allows remote and non-invasive determination of the temperature and content of the exhaust gases of various processes, engines or leaks from installations (Fig. 1). Each type of remote measurements requires different methods for quantitative interpretation of data. The direct problem in radiative energy transfer is a calculation of the spectrum of a radiating gas from knowledge of its content and temperature. It is then necessary to match the spectrum with the spectral resolution of the measurement. This means a convolution of the spectrum obtained from radiation modelling by the spectrometer apparatus function. As shown in Figs. 2 and 3, for a uniform layer of $\mathrm{CO}_{2}$ the spectrum varies significantly under the influence of both temperature and gas content. The various wavelengths of the observed spectra are substantially correlated with each other. In addition, the relationship between the spectra measurements and the determined quantities is nonlinear. In general, the spectrum of a heterogeneous gas plume is calculated with the layer model. In the simplest case, the model is composed of two layers. The analysed spectral range was proposed for 


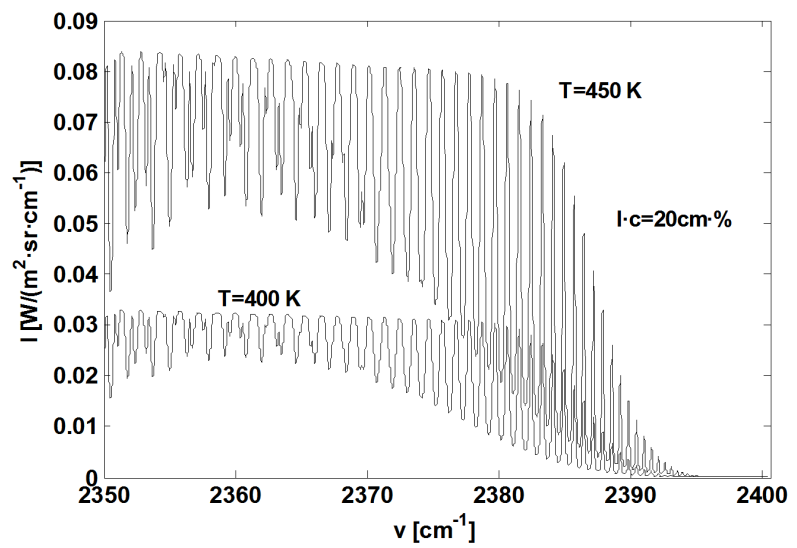

Fig. 2. Radiation spectrum of a homogeneous layer of $\mathrm{CO}_{2}$ with the content of $20 \mathrm{~cm} \%$ at two temperatures $(T=400 \mathrm{~K}$ and $T=450 \mathrm{~K})$.

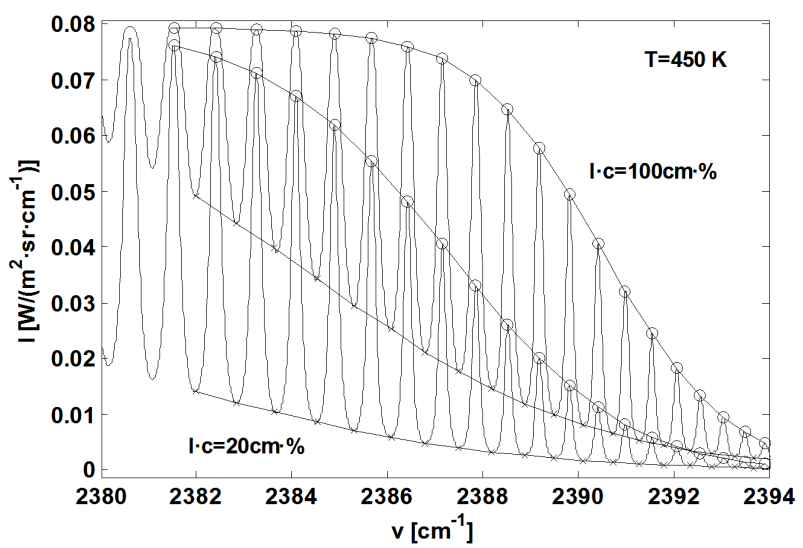

Fig. 3. Radiation spectrum of a homogeneous layer of $\mathrm{CO}_{2}$ at a temperature of $T=450 \mathrm{~K}$ for two gas contents.

determining the temperature in [43]. Figure 4 shows a radiation spectrum of the gas environment consisting of two layers: first at the temperature of $450 \mathrm{~K}$ and second at the temperature of $300 \mathrm{~K}$. This is a case often encountered in practice when the gas plume is observed from a distance. The radiation spectrum of the observed layer $(450 \mathrm{~K})$ is then absorbed by the gases from the atmosphere $(300 \mathrm{~K})$ between it and the spectrometer. The analysis of Fig. 4 shows directly that for the determination of temperature and content only the spectral range of $2370-2390 \mathrm{~cm}^{-1}$ may be used. The remainder of the spectrum is attenuated by atmospheric air between the radiating layer and the spectrometer. If this phenomenon is not taken into account, it can cause significant errors in data interpretation.

Figure 5 shows the 20 selected peaks and troughs of the spectrum, used to create spectral intensity ratios, which are then used as input for the neural network model. Each peak is divided by the nearest trough lying on its right side. In this way, a 20-element input vector of the neural model is formed.

The vector of the parameters necessary to determine the radiation can be summarised as follows:

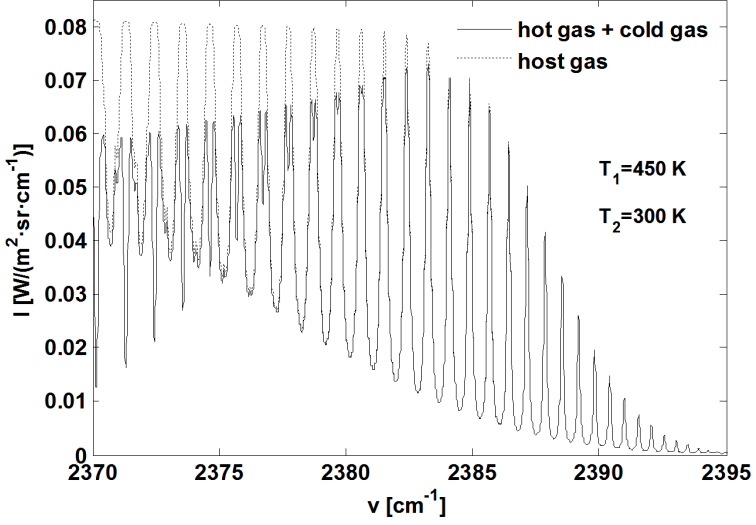

Fig. 4. Radiation spectrum of a layer of $\mathrm{CO}_{2}$ at a temperature of $T=450 \mathrm{~K}$, absorbed by a layer of temperature $T=300 \mathrm{~K}$.

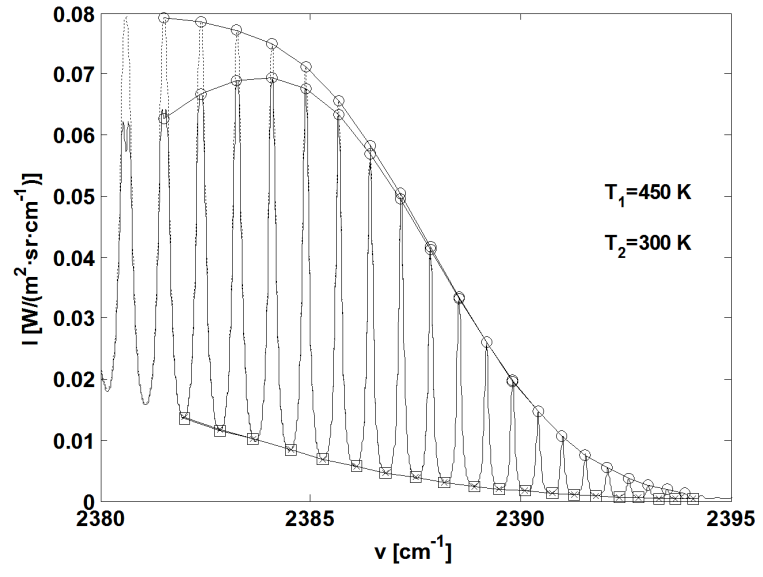

Fig. 5. Definition of peaks and troughs used to determine the ratios of radiation intensity.

$$
\boldsymbol{x}=\left(c_{1}, c_{2}, \ldots, c_{N}, t_{1}, t_{2}, \ldots, t_{N}\right),
$$

where $c_{i}$ is the content of a gas layer while $t_{i}$ is the temperature of the layer. The direct problem can be represented as a function

$$
\boldsymbol{y}=f(\boldsymbol{x}),
$$

where $\boldsymbol{y}$ is a vector of the radiation spectrum, and the function is a numerical representation of the radiative transfer equation. The inversion problem is to find the matrix $\boldsymbol{x}$ of the spectrum $\boldsymbol{y}$, with some possible additional knowledge about the phenomenon

$$
\boldsymbol{x}=f^{-1}(\boldsymbol{y}) \text {. }
$$

Unfortunately, explicit form of the inverse function cannot be determined on the basis of physical theories. Direct modelling (Eq. (2)) uses dependences given in the form of physical laws to produce synthetic measurement data. A typical procedure for solving the inverse problem involves a comparison of observations and spectra from the direct model, taking into account a certain measurement error. Adjustment of both vectors occurs by using the optimisation algorithm. Completion of the iterative 
process occurs when the difference is smaller than a specific defined value. The most frequently used methods of inversion are procedures based on the minimisation of a specific target function depending on the sought quantity $\boldsymbol{x}$ :

$$
O(\boldsymbol{x})=\sum_{i=1}^{N}\left(\boldsymbol{y}_{\text {mes }}-f(\boldsymbol{x})\right) .
$$

Since the task of this type is an ill-posed problem, all kinds of regularisation is used to obtain a well-behaved one [44]. This type of method using iterations are also called model-based inversion. In order to locate the global extremum, gradient-based or heuristic methods are applied. Grid search type algorithms (for problems with low dimensionality), simulated annealing or genetic algorithms do not require calculation of derivatives. The space of possible solutions to the inverse problem can be reduced by additional a priori knowledge. The parameter vector determined should be physically justified and limited as to the maximum and minimum values.

An alternative method of determining the sought-for quantities on the basis of measurements is the use of statistical methods in the form of parametric or nonparametric function approximation [45]. The inverse model of the estimation of the vector of the sought values of $\boldsymbol{x}$ on the basis of measurements $\boldsymbol{y}_{\text {mes }}$ can generally be written as

$$
\boldsymbol{x}=g\left(\boldsymbol{y}_{\mathrm{mes}}, \boldsymbol{a}\right),
$$

where $\boldsymbol{a}$ is the vector of the model parameters.

This solution is treated (understood) as a surrogate modelling of the estimation of the inverse function on the basis of available data [46-49]. If we have a sufficiently large set of data representative of the observed phenomenon, a statistical inverse relationship (Eq. (5)) can be built on the basis of measurements. Gathering the relevant data is not possible in all inverse problems. The inverse operator can then be trained on the basis of simulations, i.e. multiple solving of the direct problem. The operator as a function expressed by Eq. (5) is an explicit operator; the method shown in Eq. (4) is an implicit operator $[50,51]$. If it is possible to build an inverse function (inverse model), then for each measurement vector a vector of the demanded values is unequivocally determined. In the case of the iterative (or optimisation) approach, the function representing the direct problem is calculated repeatedly. In addition, in the iterative gradient methods a derivative function must be determined at each iteration. Therefore, in the case of direct physical models of complex structure and high numerical requirements, construction of simplified models is used to represent not only the transfer function but also various derivatives. This is to significantly accelerate the iterative process. Sometimes a simplified model can directly be based on empirical data. They can be used to determine the coefficients of the model based on physical theories, or to build a purely empirical model. The data set can also come from simulation. It can be used to build a direct as well as an inverse model. So the inverse algorithm (retrieval algorithm) can be based on the inversion procedure, using a direct model (Eq. (4)), or on building a inverse model based on a data set (Eq. (5)). The numerical operator determining the sought quantities using a direct model does not have an overt form. The statistical model in the form of Eq. (5) assumes the existence of an explicit form of the inverse operator. A mathematical model of a particular form must be selected here, in which on the basis of the data the sought quantities are calculated. Inverse models as well as direct, data-based ones can be built using regression methods that require assumptions relating to pre-form projection or may be based on the universal non-linear approximation such as neural networks. The method based on empirical data is used in a few cases, but especially when the physical model of a phenomenon (or process) is not available or too complicated, particularly due to a large number of parameters which are difficult or impossible to determine.

If the data for the inverse model building are synthetically generated by modelling, such methods may be called hybrid ones. The basic problem of explicit inverse models is the inability to give the parameters of such models a physical sense. This is rather reserved for models based on physical laws. In the case of data-based models, giving their parameters a physical sense is in principle only possible for linear cases. In the case of nonlinear models using a universal non-linear approximation such as neural networks we are dealing with black-box models. For such methods it is important for the learning data to include the expected range of conditions. Besides them, the properties of the method may be very weak.

\section{Method of radiation intensity ratios at multiple wavelengths}

Building statistical models of a phenomenon is preceded by the choice of input data as well as proper transformation about them. Creating a model always requires gathering a maximum of knowledge about the object. Creating a data-based model consists of the learning process that seeks the relationship between the input and output quantities. These relationships are often called mappings. What is most commonly understood here is both the search for the structure of the model and its parameters. Such a process for the artificial neural network algorithms is referred to as training.

For remote spectral measurements input data is the absolute intensity of radiation for each wavelength. Therefore, the primary requirement for correct remote measurements is absolute radiometric calibration of the measuring instrument. The study [52] shows the possibility of using the ratios of the intensity of several spectral lines to determine axisymmetric temperature profiles. It was assumed that the gas content is uniform throughout the path and contained within the specified limits. Rationing techniques do not require the advanced method of radiometric calibration. In the method of ratios we are interested only in the relative intensity of radiation for each wavelength. 


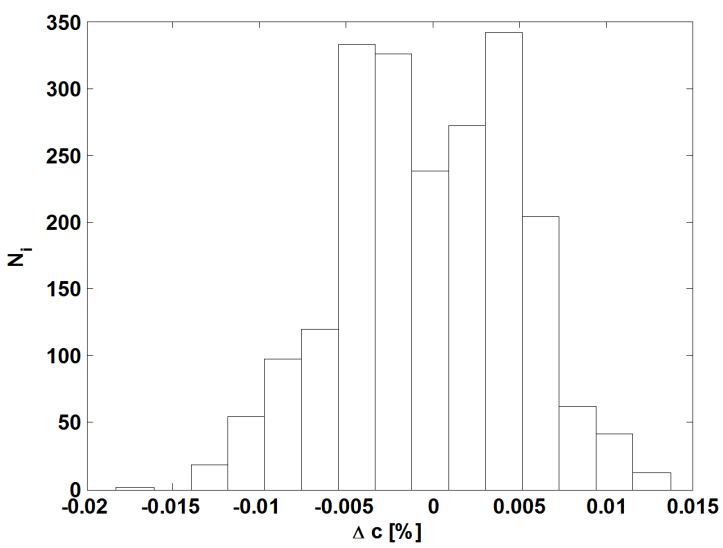

Fig. 6. Histogram of mapping errors of the $\mathrm{CO}_{2}$ content for an inverse model.

For purposes of the numerical analysis of the issue a simulated data was prepared using HITEMP database [53]. The data set comprising 2121 samples of temperatures from 350 to $450 \mathrm{~K}$ and a content of between $1 \%$ and $5 \%$ $\mathrm{CO}_{2}$ at $20 \mathrm{~cm}$. The collection was divided into a learning, validating and testing part. The first neural model was constructed to determine the gas content. A model of a multilayer perceptron with one hidden layer was used. A process of learning and testing the networks was repeatedly made with different initial values of the weights in the individual layers. The learning process was repeated for different configurations of the network with the number of neurons in the hidden layer between 4 and 10 . For 10 neurons the value of the mean square error was $3.5 \times 10^{-4 \%}$, for 4 neurons this value was $0.03 \%$. An example of error distribution for a network of 6 neurons is presented in the form of a histogram in Fig. 6. As optimal can be regarded the models of 6-8 neurons, whose mean square error oscillates around the value of $0.001-0.005 \%$.

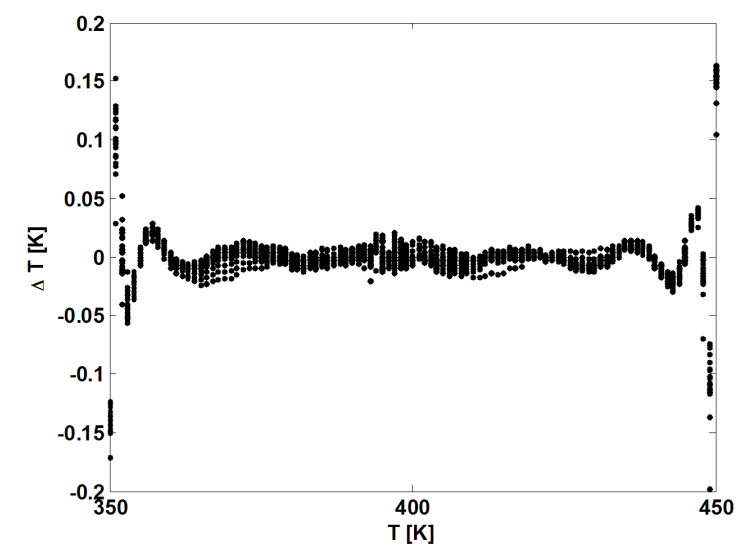

Fig. 7. The absolute error of temperature mapping for an inverse model.

A similar procedure of neural model construction was carried out for determining the temperature. The value of the mean square error for the model with 8-10 neurons for the entire data set was $0.015-0.027 \mathrm{~K}$. For $4-7$ neurons the mean square error was $0.03-0.06 \mathrm{~K}$. Figures 7,8

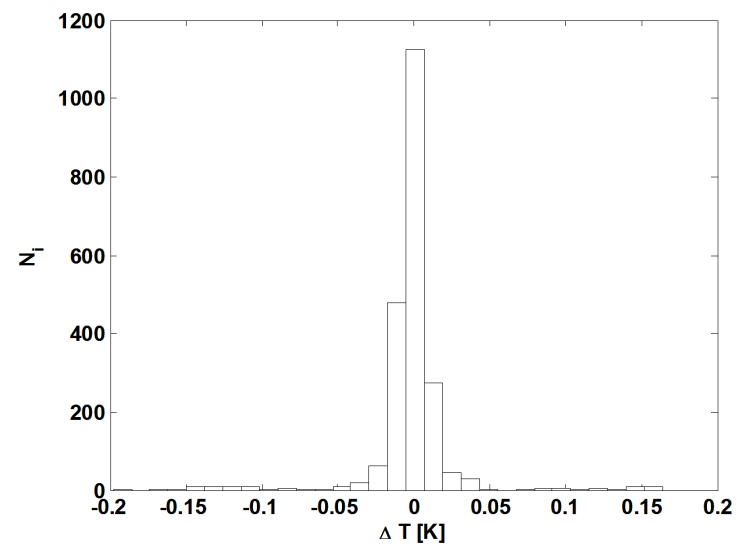

Fig. 8. Histogram of the relative error for temperature mapping.

present the characteristics of errors of the neural model for 8 neurons in the hidden layer. Distribution of absolute errors for the entire range of temperatures is shown in Fig. 7. A histogram of absolute errors is shown in Fig. 8. As can be seen, errors greater than $0.05 \mathrm{~K}$ occur only at the ends of the compartment under consideration and do not exceed $0.2 \mathrm{~K}$.

\section{Conclusions}

The paper presents the problem of determining information based on remote spectral measurements as an inverse problem of the interpretation of indirect measurements. This approach uses the knowledge of the physical foundations of the issue in question, as well as mathematical modelling. The study demonstrated the possibility of building a model mapping temperature and $\mathrm{CO}_{2}$ content based on simulation data and using a radiation spectrum in the range of $2380-2395 \mathrm{~cm}^{-1}$. It proposed a new type of transformation of the spectrum and then using it as an input vector of the model. The transformation involves the use of intensity ratios at several wavelengths. This is a new method of spectrum parametrisation, which also reduces the dimensionality of the input data. From the measuring perspective the biggest advantage of this method is to eliminate the problem of radiometric calibration. From the numerical perspective the method is characterised by a reduction in the complexity of the inverse algorithm.

\section{References}

[1] C. Oppenheimer, P. Francis, M. Burton, A.J.H. Maciejewski, L. Boardman, Appl. Phys. B 67, 505 (1998).

[2] C. Oppenheimer, P.R. Kyle, J. Volcanol. Geotherm. Res. 177, 743 (2008).

[3] M.R. Rhoby, D.L. Blunck, K.C. Gross, Opt. Expr. 22, 21600 (2014).

[4] J.L. Harley, B.A. Rankin, D.L. Blunck, J.P. Gore, K.C. Gross, Opt. Lett. 39, 2350 (2014). 
[5] O. Egorov, O. Voitsekhovskaya, D. Kashirskii, R. Tsvyk, V. Sazanovich, M. Sherstobitov, J. Quant. Spectrosc. Radiat Transf. 147, 38 (2014).

[6] G. Zhang, J. Liu, Z. Xu, Y. He, R. Kan, Appl. Phys. B 122, 2 (2016).

[7] X. Zhou, J.B. Jeffries, R.K. Hanson, Appl. Phys. B Lasers Opt. 81, 711 (2005).

[8] X. An, A.W. Casweel, J.J. Lipor, S.T. Sanders, Appl. Spectrosc. 69, 464 (2012).

[9] X. Liu, J.B. Jeffries, R.K. Hanson, K.M. Hinckley, M.A. Woodmansee, Appl. Phys. B 82, 469 (2006).

[10] K. Wu, F. Li, X. Cheng, Y. Yang, X. Lin, Y. Xia, Appl. Phys. B 117, 659 (2014).

[11] T. Cai, G. Gao, M. Wang, G. Wang, Y. Liu, X. Gao, Appl. Phys. B 118, 471 (2015).

[12] L. Ma, X. Li, W. Cai, S. Roy, J.R. Gord, S.T. Sanders, Appl. Spectrosc. 64, 1274 (2010).

[13] M.P. Szczepanski, A.W. Fountain, Appl. Spectrosc. 54, $197(2000)$

[14] S. Usseglio, K. Thorshaug, A. Karlson, I.M. Dahl, C.J. Nielsen, K.-J. Jens, E. Tangstad, Appl. Spectrosc. 64, 141 (2010).

[15] M. Strojnik, G. Paez, Opt. Expr. 23, A1259 (2015).

[16] H.J. Nam, O.J. Kwon, Infrared Phys. Technol. 67, 283 (2014).

[17] T. Fleckl, H. Jager, I. Obernberger, J. Phys. D Appl. Phys. 38, 3138 (2002).

[18] T. Ren, M.F. Modest, J. Thermophys. Heat Transf. 90, 1178 (2015).

[19] F. Onofri, M. Krzysiek, S. Barbosa, V. Messager, K.F. Ren, J. Mroczka, Appl. Opt. 50, 5759 (2011).

[20] F. Onofri, M. Krzysiek, J. Mroczka, Opt. Lett. 32 2070 (2007).

[21] J. Mroczka, D. Wysoczański, Opt. Eng. 39, 763 (2000).

[22] M.M. Li, W. Guo, B. Verma, K. Tickle, J. O'Connor, Neural Comput. Appl. 18, 423 (2009).

[23] S. Yin, S.X. Ding, X. Xie, H. Luo, IEEE Trans. Industr. Electron. 61, 6418 (2014)

[24] E. Garcia-Cuesta, I.M. Galvan, A.J. de Castro, Eng. Appl. Artific. Intellig. 21, 26 (2008).

[25] H. Schiller, Neural Networks 20, 479 (2007).

[26] M. van der Baan, C. Jutten, Geophysics 65, 1032 (2000).

[27] P. Ramuhalli, L. Udpa, S.S. Udpa, J. Appl. Phys. 93, 8274 (2003).

[28] J. Ravi, Y. Lu, S. Longuemart, S. Paoloni, H. Pfeiffer, J. Thoen, C. Glorieux, J. Appl. Phys. 97, 014701 (2005).

[29] E. Garcia-Cuesta, A.J. de Castro, I.M. Galvan, F. Lopez, Appl. Spectrosc. 68, 900 (2014).
[30] G. Świrniak, G. Głomb, J. Mroczka, Appl. Opt. 53, 4239 (2014).

[31] K. Skorupski, J. Mroczka, N. Riefler, H. Oltmann, S. Will, T. Wriedt, J. Quant. Spectrosc. Radiat Transf. 119, 53 (2013).

[32] A. Hijazi, S. Sachidanandan, R. Singh, V. Madhavan, Meas. Sci. Technol. 22, 025106 (2011).

[33] T. Hieta, M. Merimaa, M. Vainio, J. Seppa, A. Lassila, Appl. Opt. 50, 5990 (2011).

[34] G. Świrniak, G. Głomb, J. Mroczka, Appl. Opt. 53, 7103 (2014)

[35] T. Girasole, J. LeToulouzan, J. Mroczka, D. Wysoczanski, Rev. Sci. Instrum. 68, 2805 (1997).

[36] J. Mroczka, M. Woźniak, F. Onofri, Metrol. Measurem. Syst. 19, 459 (2012)

[37] J. Mroczka, Measurement 46, 2896 (2013).

[38] Z.Y. Ran, B.G. Hu, Neurocomputing 127, 88 (2014).

[39] X. Fan, M. Kang, E. Heuvelink, P. de Reffye, B. Hu, Ecolog. Model. 312, 363 (2015).

[40] A. Cozad, N.V. Sahinidis, D.C. Miller, Comput. Chem. Eng. 73, 116 (2015).

[41] P. Czop, G. Kost, D. Sławik, G. Wszołek, J. Achiev. Mater. Manufact. Eng. 44, 179 (2011).

[42] T. Girasole, G. Gouesbet, G. Grehan, J.N. LeToulouzan, J. Mroczka, K.F. Ren, D. Wysoczanski, Particle Particle Syst. Character. 14, 211 (1997).

[43] P. Al Khoury, G. Chavent, F. Clements, P. Herve, Inverse Probl. Sci. Eng. 13, 219 (2005).

[44] T. Ren, M.F. Modest, A. Fateev, S. Clausen, J. Quant. Spectrosc. Radiat. Transf. 151, 198 (2015).

[45] I.A. Kruglov, O.A. Mishulina, J. Comput. Syst. Sci. Int. 52, 503 (2013)

[46] N.V. Queipo, R.T. Haftka, W. Shyy, T. Goel, R. Vaidyanathan, P.K. Tucker, Progr. Aerospace Sci. 41, 1 (2005).

[47] J.A. Scales, R. Snieder, Geophysics 65, 1708 (2000).

[48] R. Snieder, Inverse Probl. 14, 387 (1998).

[49] G. Świrniak, J. Mroczka, J. Opt. Soc. Am. A Opt. Image Sci. Vision 33, 667 (2016).

[50] V. Cherkassky, V. Krasnopolsky, D.P. Solomatie, J. Valdes, Neural Networks 19, 113 (2006).

[51] V.M. Krasnopolsky, H. Schiller, Neural Networks 16 321 (2003).

[52] S. Cięszczyk, Proc. SPIE 9506, 950623 (2015).

[53] L.S. Rothman, I.E. Gordon, R.J. Barber, H. Dothe, R.R. Gamache, A. Goldman, V.I. Perevalov, S.A. Tashun, J. Tennyson, J. Quant. Spectrosc. Radiat. Transf. 111, 2139 (2010). 